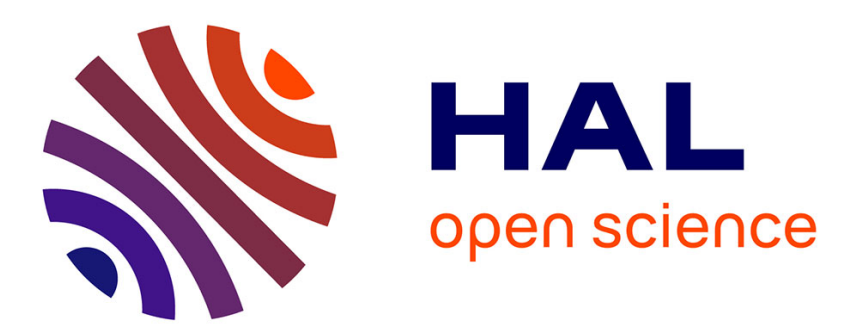

\title{
Apports et limites de la thermographie aérienne comme outil de diagnostic de la performance énergétique d'un parc résidentiel
}

Nathalie Molines, Carine Henriot

\section{- To cite this version:}

Nathalie Molines, Carine Henriot. Apports et limites de la thermographie aérienne comme outil de diagnostic de la performance énergétique d'un parc résidentiel: Exemple de l'agglomération de Compiègne.. Revue Internationale de Géomatique, 2017, 27 (1), pp.37-63. 10.3166/rig.2017.00020 . hal-01865048

\author{
HAL Id: hal-01865048 \\ https://hal.science/hal-01865048
}

Submitted on 30 Aug 2018

HAL is a multi-disciplinary open access archive for the deposit and dissemination of scientific research documents, whether they are published or not. The documents may come from teaching and research institutions in France or abroad, or from public or private research centers.
L'archive ouverte pluridisciplinaire HAL, est destinée au dépôt et à la diffusion de documents scientifiques de niveau recherche, publiés ou non, émanant des établissements d'enseignement et de recherche français ou étrangers, des laboratoires publics ou privés. 


\title{
Apports et limites de la thermographie aérienne comme outil de diagnostic de la performance énergétique d'un parc résidentiel.
}

\author{
Exemple de l'agglomération de Compiègne.
}

\author{
Nathalie MOLINES, Carine HENRIOT \\ Laboratoire AVENUES (EA 7284) \\ Département Génie des Systèmes Urbains \\ SORBONNE UNIVERSITES - UNIVERSITE DE TECHNOLOGIE DE \\ COMPIEGNE \\ Centre Pierre Guillaumat, CS 60319 - 60203 COMPIEGNE cedex \\ Nathalie.Molines@utc.fr ; Carine.Henriot@utc.fr
}

\begin{abstract}
RESUME. D'après l'ADEME (Agence de l'Environnement et de la Maîtrise de l'Energie) une toiture mal isolée peut être à l'origine de 25 à $30 \%$ des déperditions thermiques d'un bâtiment. Sachant que le parc immobilier d'une commune est majoritairement constitué de bâtiments anciens, il est fondamental d'engager une démarche globale d'amélioration de l'efficacité thermique des toitures.
\end{abstract}

Dans cette optique, l'Agglomération de la Région de Compiègne (ARC), accompagnée par l'ADEME, a mis en place une vaste campagne d'information et de sensibilisation de ses habitants et de ses élus sur l'isolation des bâtiments. Un des points d'entrée de cette campagne est un relevé thermographique aérien effectué sur l'ensemble de l'ARC. Un salon de la thermographie a été organisé par l'agglomération pour aider les habitants à interpréter les résultats de leur habitation et les informer des aides de toutes formes auxquelles ils peuvent éventuellement prétendre.

Pour aller au-delà de la simple gestion individualisée du parc bâti, l'ARC a confié au département du Génie des Systèmes Urbains de l'Université de Technologie de Compiègne une étude complémentaire. Nous présenterons dans cet article le travail effectué à l'échelle du parc bâti. L'objectif était de proposer un outil de diagnostic global du territoire et une localisation des zones à enjeux.

Deux points seront particulièrement abordés dans cet article : d'une part la mise en place d'un jeu de données permettant l'analyse spatiale, et d'autre part la création d'une suite d'indicateurs permettant d'affiner l'analyse en relativisant les déperditions thermiques par le toit. 
38 RIG. Volume $27-\mathrm{n}^{\circ} 1 / 2017$

ABSTRACT. According to the French Environment and Energy Management Agency (ADEME), a poorly insulated roof can be the cause of 25 to $30 \%$ of heat loss from a building. Given that a municipality's real estate consists mainly of old buildings, a comprehensive approach is essential to improve roof thermal efficiency.

In this context, the Urban Region of Compiègne (URC), in partnership with ADEME, has implemented an extensive public awareness campaign on building insulation, targeted at local residents and elected officials. Among other initiatives, the campaign conducted an aerial thermographic survey of the entire URC. The urban region of Compiègne organized a Thermography conference to help residents interpret data about their own residence and to inform them about relevant financial aid and other programs.

To extend the study beyond an analysis of individual buildings, the URC asked the Department of Urban Systems Engineering of the University of Technology of Compiègne to perform additional studies. This article presents the work we did for building stock.

The scope of the study was to propose a tool to conduct a comprehensive diagnostic of the territory of Compiègne and identify areas of particular interest.

This article will discuss two points: first the implementation of a database for spatial analysis, and second a set of indicators that allow indicators to refine the analysis relativizing heat loss through the roof and to identify priority intervention areas.

MoTS-CLES : morphologie urbaine, thermographie aérienne, déperdition de chaleur, SIG, indicateur, règlementation thermique, politique énergétique locale, Compiègne.

KEYWORDS: urban morphology, aerial thermography, waste heat, GIS, indicator, local energy policy, thermal building regulation, Compiègne

DOI:10.3166/rig.2017.00020 @ 2017 Lavoisier 


\section{Introduction}

Faisant suite au protocole de Kyoto, la France s'est engagée à diminuer ses émissions de gaz à effet de serre par 4 : le facteur 4 (MEDDE, 2013). Pour répondre à cet engagement, l'état français a mis en place des dispositifs législatifs et réglementaires afin que l'ensemble de ses collectivités territoriales se mobilisent pour participer à la réduction des émissions de gaz à effet de serre (Chanard, 2011 ; Chanard et al., 2011). Cette territorialisation de l'action énergétique (Theys et Vidalenc, 2011; Chanard, 2011; Godinot, 2011; Bertrand et Rocher, 2011 ; Poupeau, 2013 ; Bertrand et Richard, 2014) doit s'appuyer sur des objectifs quantifiés (Godinot, 2011) et une action territoriale globale selon trois niveaux d'intervention publique : l'exemplarité de son patrimoine et de ses services, les politiques publiques et la sensibilisation (Chanard et al., 2011). Toutefois, à l'échelle locale, l'action publique territoriale se heurte à sa propre mise en place, en raison de la difficulté de travailler sur l'efficacité énergétique réelle des formes urbaines à l'échelle d'une ville - et non simplement d'un bâtiment ou d'un îlot (Maïza, 2007 ; Arantes et al., 2016). Se positionnant à l'interface des politiques énergétiques locales et de la recherche-action sur la performance énergétique et les formes urbaines, cette contribution s'appuie sur le diagnostic énergétique de la thermographie aérienne et sur l'outil SIG pour proposer une analyse globale d'un territoire urbain.

Dans l'Agglomération de la Région de Compiègne (ARC), les émissions de gaz à effet de serre (EGES) sont de 8,1 tonnes équivalent $\mathrm{CO} 2$ par habitant et par an, se révélant ainsi supérieures aux moyennes nationale (5,5 tonnes en 2010) et régionale (7,6 tonnes pour la Picardie $)^{1}$. Or l'objectif fixé par le Grenelle de l'environnement pour 2050 est de ne pas dépasser 2 tonnes équivalent CO2 par habitant et par an. Dès lors, l'ARC a engagé son territoire vers une réduction des EGES. D'après un bilan carbone, $30 \%$ des émissions GES de l'ARC proviennent de l'habitat et du tertiaire. Or le chauffage représente la majeure partie des émissions de gaz à effet de serre des bâtiments. D'après l'ADEME, une toiture mal isolée peut être à l'origine de 25 à $30 \%$ des déperditions thermiques d'un bâtiment (ADEME, 2015). Sachant que le parc immobilier d'une commune est majoritairement constitué de bâtiments anciens, il est fondamental d'engager une démarche globale d'amélioration de l'efficacité thermique des toitures.

Dans cette optique, L'ARC, accompagnée par l'ADEME, a mis en place une vaste campagne d'information et de sensibilisation de ses habitants et de ses élus sur l'isolation des bâtiments. Un des points d'entrée de cette campagne est un relevé thermographique aérien effectué sur l'ensemble de l'ARC. Pour aller au-delà de la simple gestion individualisée du parc bâti, l'ARC a confié au département du Génie des Systèmes Urbains de l'Université de Technologie de Compiègne une étude complémentaire, laquelle s'appuie sur une méthodologie d'analyse globale des performances énergétiques, basée sur le traitement SIG des résultats de la thermographie aérienne.

\footnotetext{
${ }^{1}$ http://www.agglo-compiegne.fr/Bilan-Carbone.aspx
} 
Nous présenterons dans cet article le travail effectué à l'échelle du parc résidentiel. L'objectif est de proposer un outil de diagnostic permettant une vision globale du territoire et une localisation des zones à enjeux en termes de priorité d'intervention. Deux points seront particulièrement abordés dans cet article : d'une part la mise en place d'un jeu de données permettant l'analyse spatiale, et d'autre part, la création d'une suite d'indicateurs permettant d'affiner l'analyse en relativisant les déperditions thermiques par le toit et de repérer des zones d'interventions prioritaires.

\section{La thermographique aérienne comme diagnostic de performance énergétique}

La thermographie permet de déterminer, à distance et sans contact, la température d'un objet. Depuis une quarantaine d'années, la thermographie aérienne infrarouge est utilisée comme un outil d'évaluation de la déperdition énergétique : un bâtiment mal isolé apparaît relativement plus chaud que son environnement construit. Cette technique a été utilisée pour la première fois aux États-Unis en réaction à la crise pétrolière de 1973, lorsque l'OPEP a doublé le prix de ses exportations et imposé un embargo aux États-Unis (Allinson, 2007). Depuis le début des années 2000, les progrès techniques ont favorisé son usage à l'échelle urbaine et à l'échelle du bâtiment.

\subsection{Le principe de la thermographie aérienne}

À l'échelle d'une ville, elle est utilisée pour connaître, pour chaque bâtiment d'un territoire donné, le niveau des déperditions de chaleur. À l'échelle des bâtiments, deux types de relevés thermographiques peuvent être envisagés : la thermographie aérienne qui se concentre sur les déperditions des toits; et la thermographie des façades qui évalue plus particulièrement les déperditions thermiques des façades et des menuiseries, pour identifier d'éventuels ponts thermiques. Pour obtenir un diagnostic complet d'un bâtiment, ces deux types de thermographie peuvent être combinés. Dans le cas de l'ARC, bien que quelques thermographies de façades aient été effectuées, c'est une campagne aérienne qui a été choisie pour évaluer le parc public et privé de l'agglomération de Compiègne.

La thermographie aérienne par scanner infrarouge est basée sur la mesure du rayonnement de l'objet (en l'occurrence, la toiture) dans le domaine de l'infrarouge (Lefebvre M., 2008). Pour cela, un avion, équipé d'une trappe photo permettant d'accueillir un scanner infrarouge, survole le territoire à relativement basse altitude (400 mètres). Cette technique permet de mesurer et d'illustrer le « flux radiatif » émis par les toitures survolées. Grâce à ces flux radiatifs, il est possible, dans certaines conditions, d'estimer l'importance des déperditions thermiques des toitures, afin de les comparer entre elles. Les mesures sont réalisées de nuit en plusieurs passages, par temps froid (température au sol inférieure à $5^{\circ} \mathrm{C}$ ), aux horaires de fonctionnement maximal du chauffage, en dehors des vacances scolaires 
et des week-ends, afin que les habitants soient présents et que les bâtiments soient chauffés, en l'absence de brouillard et de neige, par temps sec et par vents faibles.

À partir de l'image thermique brute obtenue par la caméra thermique embarquée, il est alors possible d'élaborer des cartes thermiques "moyennées" ou "détaillées" en les superposant aux plans de villes ou aux plans cadastraux. La carte complète reste cependant utile pour évaluer les effets de bords ainsi que pour identifier une mauvaise superposition des couches ou une absence de mise à jour du cadastre. La carte moyennée ne reprend que la moyenne des couleurs sur la toiture. Elle permet d'obtenir des cartes moins lourdes pour la mise en ligne, de faciliter l'analyse générale en attirant l'attention sur les fortes déperditions, mais ne permet pas d'effectuer des analyses précises.

Toutefois, la thermographie aérienne ne permet pas une quantification absolue de l'énergie perdue par les toitures, et nécessite l'élaboration d'un certain nombre d'hypothèses pour permettre l'interprétation des images. Cette approche qualitative reste toutefois parfaitement pertinente dans le cadre de la sensibilisation aux économies d'énergie (Henry et al., 2009 ; Lefebvre, 2008 ; LNE, 2015).

\subsection{Etude comparative des usages de la thermographie aérienne}

Début 2016, environ 10\% des zones habitées en France ont fait l'objet d'une thermographie aérienne, ce qui représente une cinquantaine de campagnes sur une étendue de $5000 \mathrm{~km}^{2}$ (LNE, 2015). La plupart des communes ou EPCI (Établissement Public de Coopération Intercommunale) ayant réalisé une thermographie de leur territoire proposent ensuite à leurs administrés de prendre connaissance des résultats, voire de rencontrer un spécialiste afin de discuter de la performance énergétique de leur habitation. Cette démarche relève d'une action publique locale de sensibilisation. Au-delà, certaines communes intègrent également la thermographie aérienne à leur SIG existant, sans analyse complémentaire, cependant que d'autres collectivités se saisissent de ces données pour les exploiter via ou non leur SIG. Cette partie présente plusieurs exemples d'utilisation de la thermographie aérienne en France, comme à l'étranger; nous les présenterons à travers une typologie de l'action territoriale globale selon trois niveaux d'intervention publique : l'exemplarité de son patrimoine et de ses services, les politiques publiques et la sensibilisation (Chanard al., 2011), auxquels s'ajoute le diagnostic sans valorisation ultérieure.

\section{a- Un diagnostic sans autre forme de valorisation.}

En France comme à l'étranger, certaines villes n'ont pas publié les résultats de leur campagne de thermographie aérienne, craignant de mauvaises critiques dues aux problèmes de terrain ou de vent rendant l'interprétation malaisée, notamment pour les non-initiés. Ces résultats sont cependant consultables sur rendez-vous 
auprès des services municipaux, comme par exemple à Courbevoie ${ }^{2}$. Toutefois, comme l'indique le retour d'expérience de la thermographie de Bordeaux, publié sur le site de l'ADEME ${ }^{3}$ « une thermographie aérienne sans plan de communication et de sensibilisation ne sert à rien. ». D'autant plus que le coût de la campagne est conséquent : $262000 € \mathrm{HT}$, hors plan de communication et sans compter la mobilisation des deux conseillers info énergie dont les postes ont été créés pour la thermographie aérienne de la Communauté Urbaine de Bordeaux. Une campagne d'exploitation des données collectées est donc nécessaire afin de valoriser l'investissement initial et de pérenniser ses résultats. Il s'agit en somme de passer d'une action ponctuelle à une démarche effective d'élaboration d'un plan d'action pour la réduction des consommations énergétiques du parc bâti public et privé.

\section{b. La sensibilisation}

A l'instar de l'ARC, beaucoup d'agglomérations ont fait le choix de présenter leurs résultats de thermographie aérienne en ligne, sous la forme d'une carte ou de documents PDF en libre accès. L'ensemble des cartes en ligne que nous avons pu consulter utilise une représentation des niveaux de déperditions moyennés, répartis sur une échelle ordinale de six couleurs.

Sur les sites internet dédiés et lors de manifestation de présentation des résultats d'une thermographie aérienne, les Espaces Info Energie français (ou équivalents étrangers comme la Maison de l'Energie en Belgique) sont systématiquement présents. Les particuliers sont invités à les contacter. Les conseils prodigués concernent l'utilisation rationnelle de l'énergie, les énergies renouvelables ainsi que les aides et les déductions fiscales existantes lorsque des travaux sont envisagés. Ce mode d'action permet aux particuliers d'être informés, gratuitement et par des structures désintéressées, sur la maîtrise de leurs consommations énergétiques. En revanche, cette méthode ne garantit pas de retombées, contrairement à l'exemple de Bruxelles, où la thermographie semble d'abord s'adresser aux particuliers. En effet, la Région Bruxelles-Capitale a fait réaliser une thermographie aérienne sur l'ensemble de son territoire en décembre $2008^{4}$. Celle-ci a été réalisée par Eurosense Belfotop S.A., une entreprise belge dédiée aux services d'information géographique et par le Laboratoire National français de Métrologie et d'Essais (LNE) - lequel a également été mobilisé à Compiègne. Une carte dynamique présentant le niveau des déperditions moyennées, selon l'échelle standard de 6 couleurs a été mise en ligne. Cette carte permet, en offrant la possibilité aux habitants de visualiser la «couleur » de leur toiture, de sensibiliser les populations. Les personnes peuvent ensuite se rapprocher de la Maison de l'Énergie, coordonnée et financée par Bruxelles Environnement, qui leur offrira un diagnostic personnalisé et un accompagnement

\footnotetext{
${ }^{2}$ http://www.ville-courbevoie.fr/vivre-a-courbevoie/cadre-de-vie/espaces-verts-etenvironnement/etudes-et-analyses-environnementales/thermographie-aerienne.htm ${ }_{4}^{3}$ http://observatoire.pcet-ademe.fr/action/fiche/40/realisation-d-une-thermographie-aerienne 4 http://www.environnement.brussels/thematiques/energie/economiser-votre-energie/pourvous-aider/thermographie-aerienne
} 
pour les travaux. L'objectif est de favoriser la réflexion individuelle par rapport aux déperditions énergétiques, pour une amélioration du parc immobilier. Le résultat est un outil d'aide à la décision permettant aux conseillers énergie d'établir des bilans personnalisés et de déterminer les actions prioritaires. Cependant, l'affichage moyenné des déperditions ne permet pas de repérer les points particuliers comme les sorties de cheminées ou les VMC. Dès lors, cet outil doit précéder un audit énergétique complet du logement. De fait, la Maison de l'énergie propose gratuitement un audit complet et assure la réalisation de trois interventions mineures directement prises en charge sans avancement des frais par les particuliers. Il s'agit donc d'actions directes à l'échelle locale, représentatives notamment d'une culture à courte distance hiérarchique, fonctionnement dit bottom-up. Bien que ce mode d'action ne soit a priori pas directement transposable en France, il représente une opportunité vers l'optimisation des dépenses énergétiques du secteur privé.

Un autre mode d'action a été repéré à Calgary, au Canada: en plus de l'orientation vers un organisme de conseil, le site présente un classement des maisons particulières selon leur niveau de déperditions. Le projet HEAT (Heat Energy Assessment Technologies) a pour origine une initiative universitaire. Comme souvent, une plateforme en ligne dédiée ${ }^{5}$ permet aux habitants de visualiser la « couleur» de leur toiture (Hay al., 2011). À l'échelle de chaque quartier, il est possible de consulter une carte moyennée des différents bâtiments. Chaque quartier et chaque bâtiment s'est vu attribué un heat score permettant aux particuliers de comparer leur déperdition de chaleur et de se positionner par rapport à leurs voisins. Le classement crée une forme de compétition entre les habitants et peut ainsi les pousser à améliorer les performances énergétiques de leur logement. Par ailleurs, une estimation directe des économies réalisables en améliorant son isolation ou en utilisant un autre type de chauffage est parfois donnée, comme dans le cas de Calgary, Canada, où pour chaque bâtiment, les six «points chauds », ou points les plus déperditifs sont identifiés, les rejets de $\mathrm{CO} 2$ annuels moyens générés par des déperditions importantes sont calculés et s'accompagnent d'un chiffrage des économies potentiellement réalisées si le propriétaire réalise des travaux d'isolation ${ }^{6}$. C'est également un exemple de démarche bottom-up, propre cette foisci à la culture nord-américaine. On retiendra la volonté d'impliquer les habitants, malgré une méthode sans doute peu adaptée au cas français.

\section{c. Les politiques publiques}

Bien que la majorité des campagnes de thermographie aérienne se soient limitées au stade de la sensibilisation, certaines municipalités ont engagé des phases d'exploitation de résultats et d'études complémentaires, dans le cadre de leur politique de développement durable.

\footnotetext{
${ }^{5} \mathrm{http} / / / \mathrm{www}$. saveheat.co/heat-scores.php

${ }^{6} \mathrm{Ce}$ chiffrage est calculé sur la base du coût moyen de chauffage au gaz naturel.
} 
Entre 2009 et 2010, l'Atelier Parisien d'Urbanisme (APUR) a mené une étude sur le segment résidentiel du bâti parisien ${ }^{7}$. L'APUR a couplé à la thermographie aérienne trois analyses complémentaires : une étude de la construction des bâtiments (suivant les techniques de construction, les formes urbaines,...), une thermographie de façade de 500 bâtiments représentatifs et une étude critique des différentes solutions techniques existantes pour les adapter au mieux au milieu parisien. Des «planches synthèses » corrélant la période de construction, la forme du bâti et la performance thermique du bâtiment et suggérant des solutions ont été réalisées. L'ensemble vise à sensibiliser les bailleurs sociaux et les copropriétaires de la capitale aux déperditions de chaleur et propose des solutions techniques pour les réduire. Les résultats de cette étude ont ensuite été approfondis dans le cadre d'analyses complémentaires de la performance thermique de logements-types.

\section{d. L'exemplarité du patrimoine bâti public}

De même à Bordeaux, faisant suite à une campagne de thermographie aérienne en 2009, la sensibilisation des administrés prend la forme d'une plateforme en ligne expliquant le principe de la thermographie aérienne et permettant aux habitants de visualiser la «couleur» de leur toiture, c'est-à-dire le niveau de déperditions moyenné, représenté sur une échelle de six couleurs, comme pour Bruxelles ${ }^{8}$. Les habitants sont ensuite invités à prendre contact avec les Espace Info Energie pour être conseillés (A'urba, 2009). La campagne de thermographie aérienne de Bordeaux fait partie intégrante du «Plan Climat de Bordeaux Métropole », qui a pour objectifs d'agir dans les domaines de l'urbanisme et de l'aménagement, des déplacements, de la réduction des consommations énergétiques et de la promotion des énergies renouvelables, pour in fine réduire les émissions de gaz à effet de serre (A'urba, 2011 ; A'urba et Alec, 2013).

Une étude approfondie, basée non pas sur les résultats de la thermographie aérienne, mais sur la modélisation 3D des dépenses énergétiques a ensuite été réalisée. Elle s'est appuyée sur une typologie du bâti en fonction de l'année de construction et de sa morphologie urbaine, selon la méthodologie développée par l'APUR'. Dans une démarche visant à réduire les consommations énergétiques liées au chauffage, un plan de priorisation pour l'extension du réseau de chauffage a également pu être réalisé (A'urba, 2009). À Bordeaux, la thermographie a donc, avant tout, été un des principaux outils pour sensibiliser la population puis développer la politique énergétique de la ville par le recours à la modélisation 3D.

\footnotetext{
${ }^{7}$ http://www.apur.org/etude/thermographie-immeubles-parisiens-0

${ }^{8} \mathrm{http}: / / \mathrm{www}$.bordeaux-metropole.fr/plan-climat/thermographie-aerienne

9 Entretien avec Bob Clément, urbaniste spécialiste environnement, chez A'urba Agence d'urbanisme Bordeaux Métropole Aquitaine, le 25 mars 2016.
} 


\subsection{La thermographie aérienne de Compiègne}

Dans le cas d'étude compiégnois, une démarche globale a été initiée par la Communauté d'agglomération de la région de Compiègne, l'ARC. Suivant la typologie de l'action publique envisagée par Chanard, de Sède-Marceau et Robert, (2011), c'est préoccupée par les dépenses énergétiques de son patrimoine bâti, pour servir d'outil de sensibilisation auprès du grand public et pour fonder scientifiquement la politique énergétique locale que l'ARC a sollicité le département du Génie des systèmes urbains de l'Université de technologie de Compiègne, dans le cadre d'un atelier projet de 19 étudiants regroupés en «bureau d'études » et encadrés par trois enseignants-chercheurs.

Une campagne de thermographie aérienne a été réalisée le 2 février 2015 sur le territoire de l'ARC par le Laboratoire National de métrologie et d'Essais (LNE). Les résultats de cette thermographie aérienne ont été publiés sur une plateforme, hébergée sur le site internet de l'ARC ${ }^{10}$, et diffusés auprès du grand public dans le cadre d'un week-end de sensibilisation aux déperditions d'énergie les 26 et 27 septembre 2015, encadré par le LNE et de quatre journées lors de la foire_exposition du Compiègnois en octobre 2015. Durant ce «salon de la thermographie », l'équipe de travail de l'atelier projet, formée durant une journée à l'interprétation des cartes thermographiques par le LNE accueillait le public désireux que lui soit interprété le résultat de son habitation. Celui-ci se voyait ensuite remettre un visuel, avant d'être orienté vers le stand du centre info énergie, pour y être informé et conseillé en termes de travaux de rénovations énergétiques de leurs habitations. Dans un second temps et durant les 4 mois qui ont suivis, le bureau d'étude constitué cherchait à valoriser davantage les résultats de l'image thermographique. La commande de l'ARC visait notamment à comprendre les relations existant entre les déperditions thermiques captées par la caméra infrarouge et d'autres éléments permettant de caractériser l'urbain tels que la forme urbaine (forme des îlots, densité de constructions...), le type de bâti (hauteur de construction, collectif, individuel...) ou l'année de construction, notamment après l'apparition des réglementations thermiques en 1974. Le second objectif de notre étude visait à dégager des zones prioritaires d'intervention pour lesquelles, après complément d'information, une mutualisation des interventions serait bénéfique. Il était également demandé une mise au point d'un outil synthétique de connaissance de l'état du parc public et la constitution d'une base de données intégrant les résultats de la thermographie aérienne, pour en faire un outil opérationnel d'aide à la décision.

L'étude de la consommation énergétique des bâtiments a fait l'objet de plusieurs travaux. En physique urbaine, les auteurs utilisent généralement, comme entrée principale, le calcul d'une consommation énergétique théorique. Celle-ci est généralement calculée en fonction de l'âge du bâtiment, prenant ainsi en considération les réglementations thermiques françaises en vigueur au moment de la construction, et de sa forme urbaine. (Maïzia, 2007 ; Kavgica et al, 2010). Dans la

\footnotetext{
10 Site internet de la carte thermique de l'ARC, http://geo.agglocompiegne.fr/thermographie/index.html
} 
poursuite de ces travaux de modélisation, Arantes et al. (2016) ont largement pondéré l'évidence de relations existantes entre compacité (urbaine), densité et performances énergétiques, pour favoriser la notion d'«optimisation morphoénergétique ». Ces travaux soulignent la difficulté de travailler sur la performance énergétique et les formes urbaines à l'échelle de la ville (Maïza, 2007 ; Arantes et $a l ., 2016)$ et préconisent d'évaluer la dépendance énergétique d'une ville, à partir de sa densité et de sa composition morphologique, à partir d'îlots et de petits secteurs, puis de généraliser ces formes urbaines à l'ensemble de la ville, dans une perspective, non pas de préconisation, mais de compréhension (Arantes et al., 2016).

Par ailleurs, l'analyse de la déperdition énergétique réelle des bâtiments à l'échelle urbaine, est, à notre connaissance, moins étudiée. Y. Ham et M. GolparvarFard (2013) mettent en avant les limites de l'utilisation de logiciels de simulation énergétique pour de tels projets - temps de main d'œuvre important, non prise en compte des défauts de construction et des dégradations, manque de précision - pour proposer un modèle 3D basé non pas sur la consommation énergétique théorique d'une forme urbaine, mais sur la thermographie de façades. Cet outil, bien que proposant une représentation réelle de l'état actuel de la performance énergétique des bâtiments, reste toutefois long, couteux et fastidieux à mettre en place à l'échelle d'une agglomération.

Au-delà de la thermographie des façades, la thermographie aérienne offre une alternative, à grande échelle, de l'évaluation de la performance énergétique réelle d'une ville. Pour autant, les articles scientifiques mobilisant la thermographie aérienne présentent souvent les étapes amont du traitement de l'image infra rouge et de son recalage avec les données cadastrales (Vellico et al., 2011) ou évaluent le micro climat urbain et les îlots de chaleurs (Vermeulen et al., 2013 ; Chandelier et al., 2014). À notre connaissance, seule la Communauté urbaine de Dunkerque semble avoir véritablement intégré la thermographie aérienne aux matériaux de construction (supposés ?) et à la consommation (supposée ou réelle ?) pour caractériser la performance énergétique de son parc bâti, sous SIG, dans le cadre du projet Anaspat RéGES devenu STARTER en $2012^{11}$. Cependant il ne semble pas que ce travail ait fait l'objet de publications scientifiques et nous ne sommes pas parvenues à entrer en contact avec l'équipe technique pour échanger quant à la méthodologie employée.

\section{Méthodologie et jeu de données}

\subsection{Méthodologie}

La méthode mise en place pour cette partie du projet a été divisée en plusieurs étapes :

\footnotetext{
${ }^{11}$ Projet STARTER porté par le Communauté urbaine de Dunkerque et présenté sur le site européen Energy Cities. http://www.energy-cities.eu/IMG/pdf/15e_atelier_a_1_action_23062015_cud.pdf
} 
- Interprétation, géoréférencement et analyse statistique de l'image thermographique. Cette étape a été effectuée par le bureau d'études LNE en amont de notre intervention;

- Création d'un jeu de données multi-sources permettant de relier les données issues de la campagne de relevé thermographique au parc bâti résidentiel et à ses différentes caractéristiques ;

- Analyse de la déperdition thermique des toits grâce à une série d'indicateurs. L'objectif est de relativiser le résultat obtenu par la campagne thermographique grâce à un indice de déperdition relative par le toit. On appelle indice de déperdition relative par le toit, le pourcentage de déperdition qui pourrait s'échapper $\mathrm{du}$ toit d'une habitation. Celui qui est utilisé dans notre modèle est très simple puisqu'il dépend uniquement de la forme du bâtiment: surface de façades et mitoyenneté. Il ne prend ni en compte une éventuelle isolation du toit ni les types de matériaux utilisés. Cet indice permet d'évaluer rapidement quelle est la part de déperdition potentielle du toit d'un bâtiment sur l'ensemble du parc résidentiel de l'agglomération (soit près de 20000 bâtiments).

- Localisation de zones d'intervention prioritaire grâce à la corrélation, dans un premier temps, de l'indice de déperdition relative et des informations produites par le relevé de thermographie aérienne. Dans un deuxième temps, des données sur le pourcentage de bas revenus dans une maille donnée sont intégrées au modèle pour définir des zones ultra prioritaires. Ces zones d'enjeux serviront de base à l'agglomération pour cibler ses campagnes d'information et de sensibilisation aussi bien auprès des particuliers que des gestionnaires de parcs locatifs.

Pour faciliter la lecture des cartes, quatre zones tests ont été sélectionnées et seront présentées dans cet article (cf Figure 1):

- Une zone d'habitats sociaux de petits et grands collectifs de la fin des années 80. Cette zone s'organise autour d'un groupe scolaire central qui a été conservé dans l'analyse (zone 1);

- Une zone de centre-ville, de tissu dense, composée de petits collectifs de 3 ou 4 étages et de maisons individuelles très souvent mitoyennes (zone 2) ;

- $\quad$ Une zone péri urbaine mixte de lotissements et de petits collectifs (zone 3) ;

- $\quad$ Une zone rurale lotie en maisons accolées et individuelles (zone 4).

Ces zones ont été sélectionnées car elles présentent des formes urbaines bien distinctes ainsi que des déperditions relativement élevées. Ces zones sont donc représentatives des formes urbaines de l'ARC mais ont des déperditions thermiques supérieures à la moyenne (cf Tableau 1). 


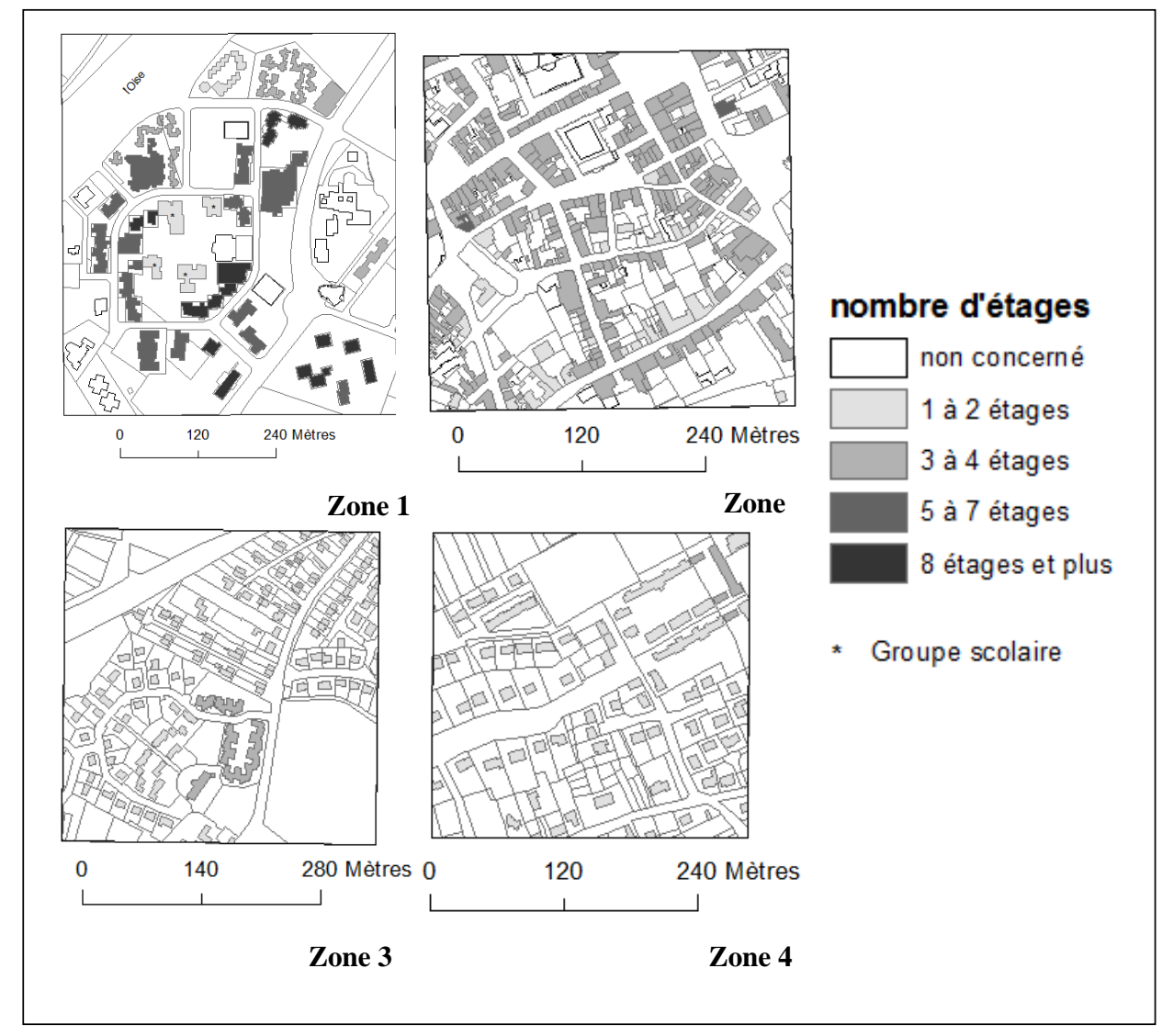

Figure 1 : présentation des 4 sites sélectionnés

Tableau 1: Caractéristiques du territoire

\begin{tabular}{|c|c|c|c|c|c|c|}
\hline Zone & $\begin{array}{c}\text { Nbre } \\
\text { d'habit } \\
\text { ations }\end{array}$ & $\begin{array}{c}\text { Surface } \\
\text { moyenne } \\
\text { au sol } \\
\left(\mathrm{m}^{2}\right)\end{array}$ & $\begin{array}{c}\text { Taux habitat } \\
\text { individuel } \\
(\%)\end{array}$ & $\begin{array}{c}\text { Taux } \\
\text { habitat } \\
\text { collectif } \\
(\%)\end{array}$ & $\begin{array}{c}\text { Nbre } \\
\text { étages } \\
\text { moyen }\end{array}$ & $\begin{array}{c}\text { Âge moyen du } \\
\text { parc bâti (nbre } \\
\text { d'années })\end{array}$ \\
\hline 1 & 48 & 811 & 0 & 96 & 5,3 & 40 \\
\hline 2 & 281 & 125 & 27,5 & 72,5 & 3 & 135 \\
\hline 3 & 132 & 101 & 91 & 9 & 2,2 & 77 \\
\hline 4 & 74 & 125 & 89,2 & 11,8 & 1,7 & 55 \\
\hline ARC & 19317 & 101 & 93,1 & 6,9 & 1,9 & 86 \\
\hline
\end{tabular}


Apports et limites de la thermographie 39

\subsection{Jeu de données multi-sources}

Une fois l'image thermographique géoréférencée et interprétée (plusieurs indicateurs sont fournis par le bureau d'études pour chaque bâtiment : pourcentage de chaque classe de déperdition, valeur moyenne de la déperdition sur 6 ou 256 classes...), un travail important est à effectuer pour rattacher cette donnée à d'autres données caractérisant le bâtiment.

Plusieurs sources de données sont ainsi associées à l'image thermographique :

- les données cadastrales, présentes sous deux formes: des matrices rassemblant un grand nombre de données liées soit aux parcelles cadastrales soit aux bâtis construits; et des couches géoréférencées comportant des informations sur le parcellaire et le bâti construit. Cette donnée va nous permettre de récupérer les principales caractéristiques du bâtiment comme l'année de construction, le nombre d'étages, le type (maison, appartement, dépendance, local commercial ou industriel), le matériau composant principalement la toiture...

- la photographie aérienne orthorectifiée et google view ont été utilisés pour vérifier que les masques correspondant à l'emprise cadastrale, utilisés par le bureau d'études pour extraire l'information concernant les bâtiments, ne comportent pas d'erreur susceptible d'entraîner une évaluation erronée du bâtiment. En effet, les surfaces végétales ou goudronnées ressortant par exemple en classe de déperdition maximale correspondent souvent à un décalage par rapport à l'emprise réelle du bâti. La présence d'un arbre surplombant un toit peut également modifier le résultat (cf Figure 2).

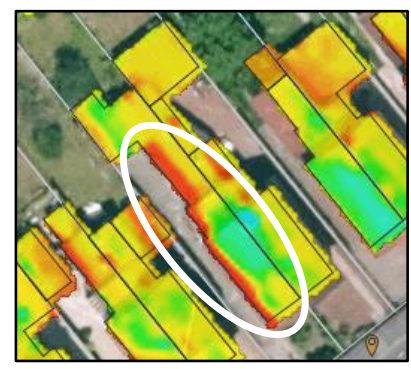

Figure 2 : Exemple de biais engendré par un décalage entre le masque bâti et l'emprise réelle du bâti

- Les données carroyées INSEE à 200 mètres proposent un certain nombre de données sociodémographiques dont le nombre total de ménages présentant un revenu fiscal par unité de consommation inférieur au seuil de bas revenu. En rapportant ce nombre au nombre total de ménages de chaque carreau, on peut comparer la situation économique des ménages de l'agglomération. 
- La BD TOPO de l'IGN ; qui servira de fond de plan de référence pour cartographier les rues et les cours d'eau.

Seules les habitations nous intéressant à cette étape, un premier travail de nettoyage de la base cadastrale a été effectué. Ont été sortis de l'analyse :

- Les bâtiments publics, commerciaux et industriels ; seul le groupe scolaire au centre de notre zone d'habitats sociaux de petits et grands collectifs a été conservé car il présente un déficit d'isolation majeur et pourrait rentrer dans une opération globale de réhabilitation du quartier.

- Les constructions légères comme les vérandas, les préaux et autres cabanons. Seul le bâti dur revêt un intérêt pour l'analyse de la thermographie aérienne : en effet, les déperditions thermiques de bâtiments non chauffés n'engendrent pas d'émissions de GES. De plus elles viendraient polluer l'analyse statistique, car leurs valeurs sont la plupart du temps extrêmes alors qu'elles ne sont pas pertinentes ;

- Les polygones de petites superficies (inférieures à $15 \mathrm{~m}^{2}$ ), ceux-ci ne pouvant pas correspondre à des habitations.

Une fois la couche nettoyée, une jointure spatiale est effectuée avec la couche thermographique. Cette jointure nous permet, grâce à la géolocalisation des deux couches, de récupérer dans la couche «Bâtiment», les attributs de la couche « thermographie ». Un certain nombre de problèmes ont été rencontrés :

- Les données cadastrales sont fragmentaires, les critères rattachés à chaque parcelle imposable ne sont pas renseignés pour toutes les parcelles ;

- Certaines données semblent incohérentes. Par exemple, l'année de construction indiquée dans la base est incohérente par rapport au visuel du bâtiment lui-même. D’autres données sont simplement fausses (année de construction en 120 ou en 3345 par exemple);

- Le limites des données thermographiques : un certain nombre d'éléments peuvent fausser l'interprétation des résultats thermographiques (Henry al., 2009). Ces biais sont d'abord liés à la méconnaissance de l'usage du bâti (combles aménagés ou non, logement chauffé ou non à la date du passage de l'avion...). Ceci peut entraîner un décalage d'une classe dans le résultat obtenu. Lorsque l'image thermographique est exploitée à des fins individuelles (information d'un propriétaire) ces incertitudes sont facilement levées et il est possible de rééchelonner le résultat. Cependant, ce n'est pas le cas lors de l'évaluation globale du territoire. Les résultats obtenus doivent donc être pris avec précaution, en gardant en mémoire que cet indice nous donne une tendance mais non la réalité exacte. Le décalage entre l'image thermographique et le cadastre (cf. figure 1) peut également entraîner une 
distorsion de la note moyennée. Le travail en amont de recalage est primordial (Allinso, 2007 ; Vellico et al, 2011)

\section{Création d'indicateurs affinant l'indice de base de déperdition énergétique par le toit}

Quatre indices ont été développés (Cf Figure 3):

- L'indice 1 exploite l'image thermographique et propose une déperdition énergétique moyenne pour chaque bâtiment ;

- L'indice 2 affine l'analyse en calculant, à partir des données cadastrales, l'indice de déperdition potentielle des toits ;

- L'indice 3 met en corrélation les deux premiers pour repérer les zones d'intervention prioritaires :

- L'indice 4 met en corrélation les zones prioritaires avec les données carroyées de l'INSEE sur les bas revenus pour en déduire les zones d'intervention ultra prioritaires.

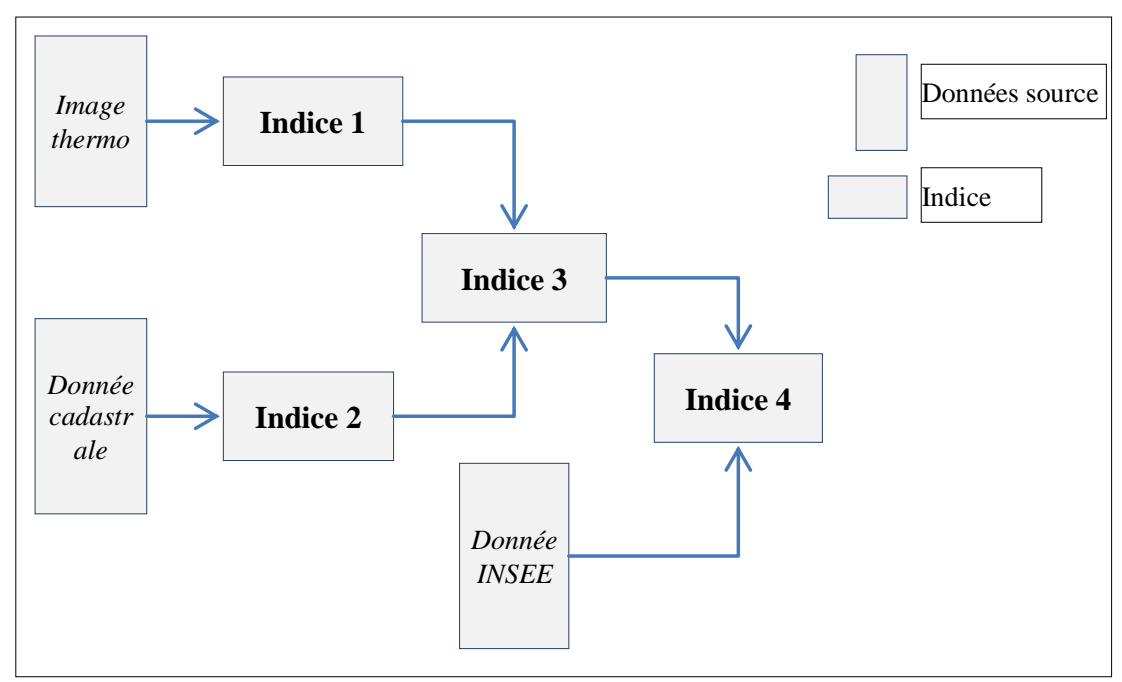

Figure 3 : méthodologie de création des indices

\subsection{Indice de déperdition moyenne des bâtiments :}

L'indice de déperdition moyenne des bâtiments a été obtenu en deux étapes. Partant de l'image aérienne à 4096 nuances de gris correspondant au rayonnement infrarouge perçu par la caméra thermique, le bureau d'études mandaté a obtenu une carte à 256 niveaux de couleurs selon une répartition gaussienne permettant de 
s'affranchir des valeurs extrêmes. Ces 256 niveaux de couleurs ont ensuite été discrétisés en 6 niveaux de façon à ce que chacune des six couleurs couvre globalement la même proportion de surface thermographiée.

Cet indice peut être facilement exploité. Il correspond à une déperdition moyenne des bâtiments. Il permet, en comparant les déperditions de l'ensemble des bâtiments, de faire ressortir les bâtiments les plus déperditifs (cf Tableau2).

Comme nous l'avons expliqué précédemment, ces résultats sont à relativiser avec les limites des données et des résultats thermographiques produits. Ils permettent cependant d'avoir une vue globale de l'agglomération. La carte produite (cf Figure 4) permet visuellement de voir se détacher plusieurs zones à enjeux. Ces secteurs correspondent à des ensembles géographiques de plus ou moins grandes superficies où la majorité des bâtiments est identifiée comme déperditive. Dans le cas où ces zones correspondent à des habitats collectifs, si la déperdition est confirmée, une action de sensibilisation et d'accompagnement pourra être menée auprès du bailleur social. Cela peut également être des lotissements composés de maisons aux systèmes constructifs identiques. On pourra alors informer les propriétaires et les guider vers des regroupements de travaux afin de réaliser des économies d'échelle.

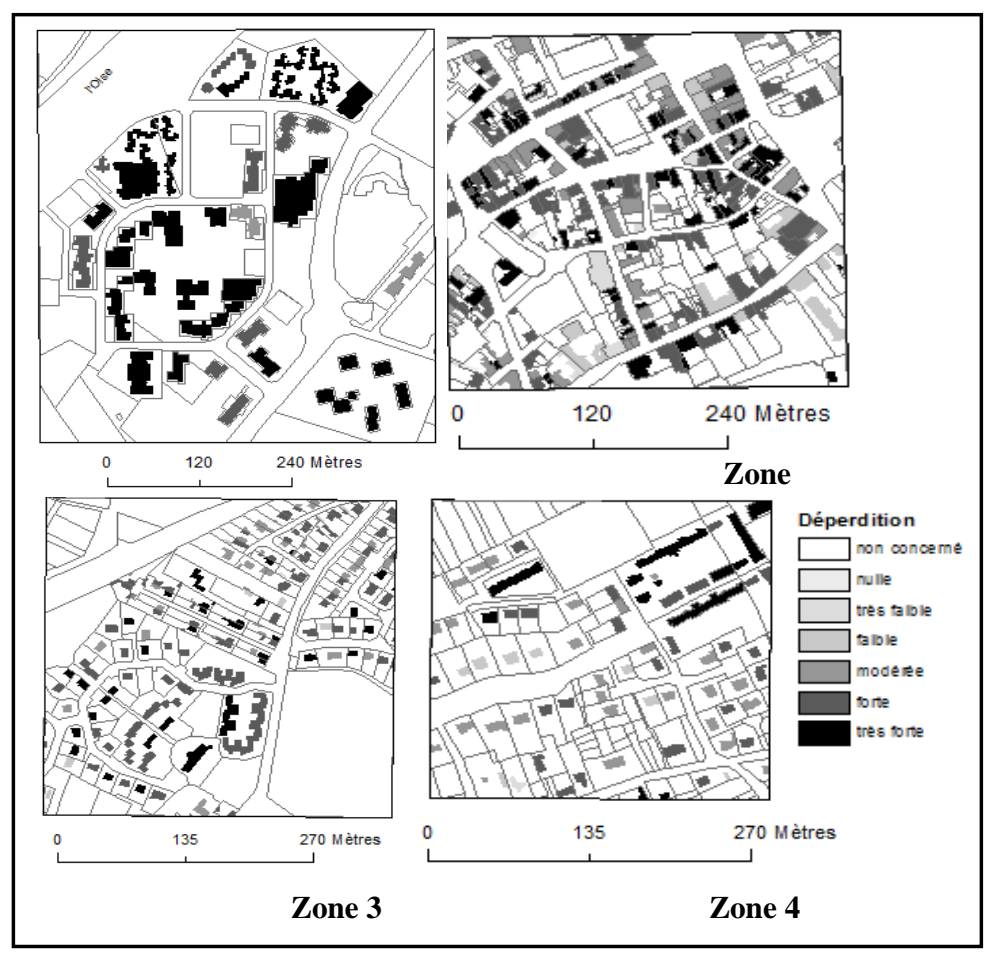

Figure 4 : Déperditions moyennes des toits 
Tableau 2 : Caractéristiques du territoire

\begin{tabular}{|c|c|c|c|}
\hline Zone & $\begin{array}{c}\text { Nbre } \\
\text { d'habitations }\end{array}$ & $\begin{array}{c}\text { Surface moyenne au } \\
\text { sol }\left(\mathrm{m}^{2}\right)\end{array}$ & $\begin{array}{c}\text { Déperdition moyenne } \\
\text { (sur 6) }\end{array}$ \\
\hline 1 & 48 & 811 & 5,7 \\
\hline 2 & 281 & 125 & 4,7 \\
\hline 3 & 132 & 101 & 5 \\
\hline 4 & 74 & 125 & 4,4 \\
\hline ARC & 19317 & 101 & 4 \\
\hline
\end{tabular}

\section{Interprétation :}

Ces quatre zones sont assez représentatives du parc résidentiel de l'agglomération compiégnoise : un parc plutôt ancien constitué en grande partie d'habitations individuelles non mitoyennes. Quelques zones d'habitations collectives en barres, en tours ou en plots viennent rompre ce paysage urbain relativement homogène.

En partie dû à l'âge de ce parc, les déperditions sont de moyennes à élevées. Certaines zones prioritaires apparaissent dès ce stade ; c'est le cas par exemple de la zone 1 avec une déperdition moyenne « très forte ».

\section{Limite de cet indice :}

Ce premier indice nous donne déjà une première idée de l'état du parc résidentiel. Cependant, il ne tient pas compte de la spécificité des différentes formes urbaines.

Cet indice se focalise en effet uniquement sur la donnée thermographique. Cependant les caractéristiques de la morphologie urbaine peuvent à elles seules diviser par deux la consommation énergétique d'un morceau de ville (Baker, 2000 ; Ratti, 2005). En effet la part de déperdition potentielle ${ }^{12}$ par le toit d'une construction ne sera pas la même pour une tour de grande hauteur que pour une maison isolée ou une habitation mitoyenne de centre-ville (MEDDAT, 2009).

Comme le montre la figure 5 , si la déperdition moyenne par le toit d'une maison de plain-pied est de près de $30 \%$ pour une maison isolée, elle sera beaucoup moins importante pour une tour de grande hauteur (dont la part de déperdition par les façades sera beaucoup plus importante) et beaucoup plus forte pour des maisons

\footnotetext{
${ }^{12}$ Une part de déperdition est toujours potentielle puisque si une paroi est isolée, elle ne sera plus déperditive.
} 
mitoyennes dont une ou deux façades ne seront pas à prendre en compte dans le total des surfaces potentiellement déperditives (Al Homoud, 2005 ; Henry al., 2009, Maresca et al, 2009).

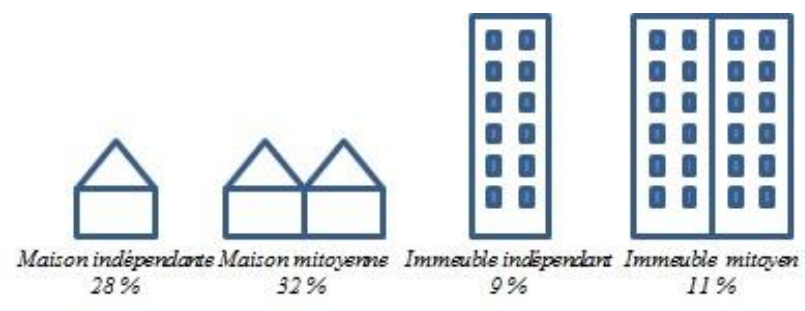

Figure 5 : Taux moyen de déperdition par le toit en fonction de la forme urbaine (d'après MEDDAT, 2009)

\section{2 : Calcul de la part potentielle des toits dans la déperdition effective d'un bâtiment}

Pour pallier cette limite, un deuxième indice est développé. Celui-ci évalue la « part potentielle des toits dans la déperdition effective d'un bâtiment ». Trois données supplémentaires sont nécessaires: la superficie des toitures, la superficie totale de façades et la superficie de façades non mitoyennes. Cet indice est indépendant des résultats thermographiques; il nous permet de repérer les bâtiments pour lesquels, si cela s'avère nécessaire, une isolation du toit fera baisser significativement les déperditions totales du bâtiment ${ }^{13}$. Il sera croisé dans un deuxième temps avec les données thermographiques.

La surface de déperdition désigne toutes les surfaces de l'enveloppe qui séparent l'intérieur de l'habitation d'un espace non chauffé. On y retrouve la toiture, le sol, et les façades non mitoyennes. L'importance du facteur mitoyenneté dans le transfert thermique des bâtiments est bien connu (Zayane, 2011) et a été confirmé lors du bilan thermique du bâti de la ville de Bordeaux (A'Urba, 2009).

Dans le cadre des surfaces mitoyennes, le flux de chaleur est quasiment nul. En effet, en cas de surfaces mitoyennes, on considère qu'il n'y a pas d'écart de température entre les deux côtés du mur, ce qui correspond à un flux de chaleur nul. Les pertes par le sol étant négligeables (moins de $10 \%$ des déperditions totales pour une maison de plain-pied, non mitoyenne et quasiment nulle pour une tour de grande hauteur), nous ne les avons pas prises en compte dans notre modèle.

13 Cela peut être intéressant à savoir pour un particulier ne disposant pas du budget nécessaire à l'isolation de la totalité de sa maison (toit, façades, fenêtres...). Cela permet également de relativiser les déperditions par le toit des bâtiments de plusieurs étages. 
Plusieurs étapes sont ensuite nécessaires :

a- Calcul de la superficie des toitures : on différencie les toits plats, dont la surface de toiture correspond à l'emprise sur le plan masse du bâtiment, des toits en pente qui ont une superficie supérieure. Un coefficient de 1,35 à $\mathrm{S}$ (l'emprise au sol du toit) est attribué aux toits en pente. Ce coefficient a été obtenu en considérant une toiture à 2 pans présentant un angle de $42^{\circ}$.

b- Calcul de la superficie de façades non mitoyennes : la superficie de parois mitoyennes est calculée à partir de la longueur des parois mitoyennes fournies par le SIG. Cette donnée nous permet de déduire la surface de façades mitoyennes

Superficie mitoyenne $=($ Longueur mitoyenne $x$ hauteur $)$

Cette équation nous fournit un indice simplifié : on considère en cas de façades mitoyennes que la totalité de la façade est mitoyenne (même si la mitoyenneté se fait avec un bâtiment de taille inférieure), les surfaces vitrées (davantage déperditives) ne sont pas prises en compte. Pour l'objectif visé, cette simplification est largement suffisante.

c- Calcul de la part potentielle des toits dans la déperdition effective d'un bâtiment :

Déperdition par le toit $=\frac{S \text { Toit }}{(S \text { Toit }+S \text { Façades })-S \text { Facades mitoyennes }} \times 100$

\section{Interprétation :}

Ce rapport permet d'appréhender une part plus réaliste des déperditions de la toiture d'un bâtiment. Plus ce rapport est grand, plus le taux de déperdition potentielle par la toiture est important. Cela ne prend néanmoins pas en compte l'isolation du bâtiment ou encore sa surface vitrée, qui doivent être étudiées pour chaque bâtiment.

Cet indice confirme que la part de déperdition potentielle par le toit peut considérablement varier en fonction de la forme du bâtiment (superficie, hauteur) et de la présence ou non de mitoyenneté. On a ainsi une déperdition potentielle par le toit de $11 \%$ pour une tour de 13 étages et une déperdition de plus de $80 \%$ pour un immeuble de centre-ville dont les façades sont mitoyennes à près de $70 \%$

Tableau 3 : Analyse statistique Indice 2

\begin{tabular}{|c|c|c|}
\hline Zone & Taux mitoyenneté $(\%)$ & Part potentielle des toits (\%) \\
\hline 1 & 0,7 & 28,1 \\
\hline
\end{tabular}




\begin{tabular}{|c|c|c|}
\hline 2 & 34 & 31,7 \\
\hline 3 & 12,5 & 35,1 \\
\hline 4 & 0,9 & 38,9 \\
\hline ARC & 11 & 36,9 \\
\hline
\end{tabular}

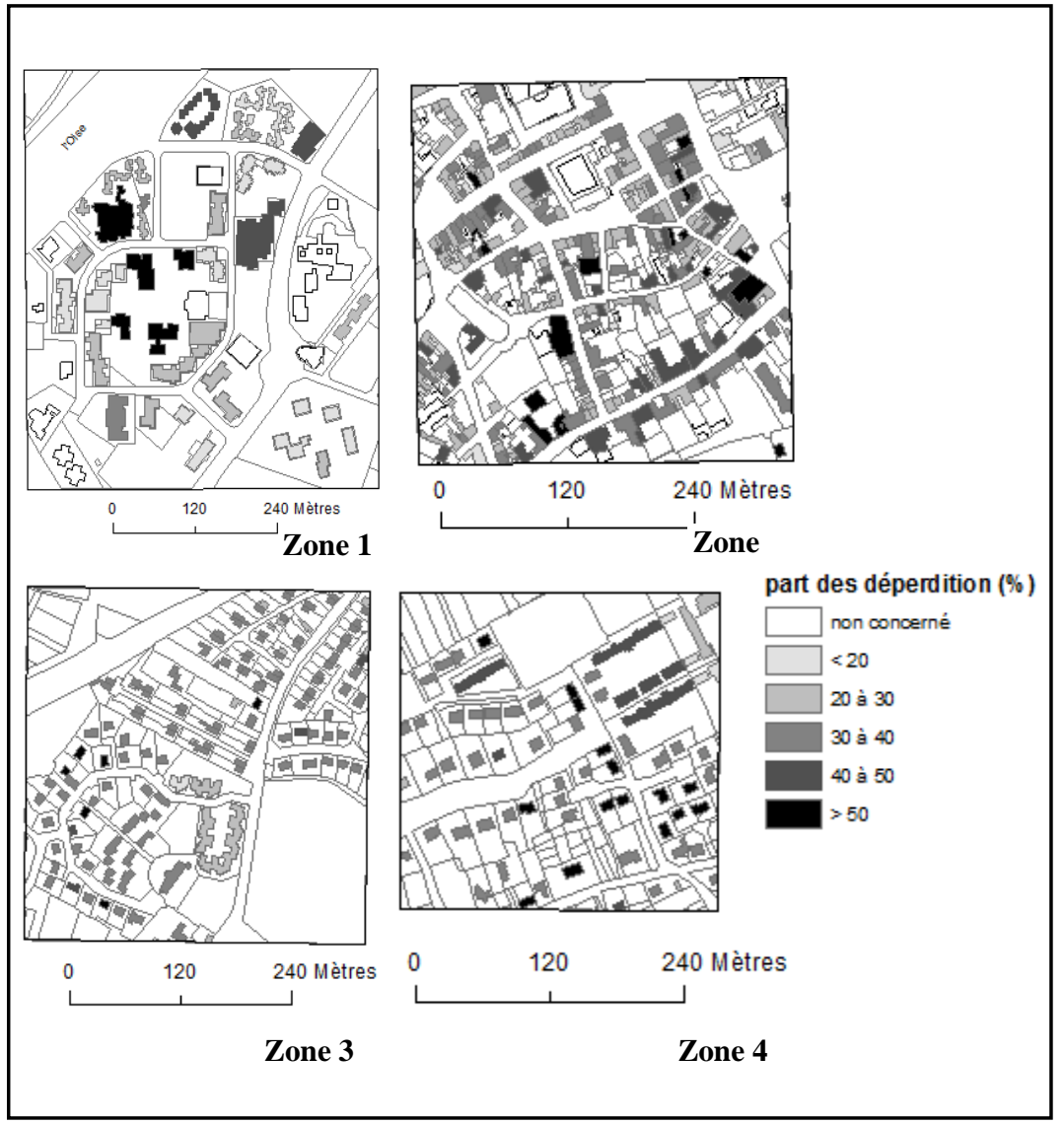

Figure 6: Part potentielle des toits dans la déperdition effective

La zone 1, constituée de plusieurs immeubles de grandes hauteurs (8 à 12 étages), possède naturellement l'indice moyen de déperdition potentielle par le toit le plus faible (cf tableau 3 et Figure 6). Malgré tout cet indice reste relativement élevé pour une zone d'habitat collectif car des bâtiments collectifs en barre (de 2 à 4 étages) et un groupe scolaire constitué de 3 bâtiments de un à deux étages sont mélangés aux grands collectifs. Si l'on soustrait le groupe scolaire de l'analyse statistique, on se retrouve avec une déperdition moyenne de $25,8 \%$. Toutes les 
autres zones ont des déperditions potentielles par le toit bien supérieures à cette valeur. La zone 2, zone dense de centre-ville avec un taux de mitoyenneté très élevé, fait également baisser la moyenne de l'agglomération.

\section{Limite de cet indice :}

Cet indice ne tient pas compte de l'isolation potentielle du toit : si un toit est bien isolé, la déperdition par le toit sera minime, et ce, même si la part de déperdition potentielle est grande. L'intégration dans le modèle des données thermographiques permet de contourner cette limite.

\section{3 : Délimitation des zones d'intervention prioritaires}

Les deux indices précédents sont mis en corrélation afin de localiser les bâtiments dont la part de déperdition potentielle par le toit est supérieure à la moyenne de l'agglomération (cf tableau 3) et dont les déperditions thermiques sont fortes ou très fortes ${ }^{14}$.

Ce nouvel indice met ainsi en avant les bâtiments dont la forme fait que la part de déperdition potentielle des toits est supérieure à la normale et dont la campagne thermographique a effectivement révélé de fortes déperditions (cf tableau 4 et figure 7).

Tableau 4 : Analyse statistique de l'indice 3

\begin{tabular}{|c|c|c|c|}
\hline Zone & $\begin{array}{c}\text { Nombre de bâtiments } \\
\text { prioritaires }\end{array}$ & $\begin{array}{c}\% / \text { total parc résidentiel de la } \\
\text { zone }\end{array}$ & $\begin{array}{c}\text { Superficie } \\
\text { concernée }\left(\mathrm{m}^{2}\right)\end{array}$ \\
\hline 1 & 11 & 36 & 13716 \\
\hline 2 & 56 & 25,3 & 8925 \\
\hline 3 & 27 & 27,7 & 3049 \\
\hline 4 & 18 & 23,0 & 3380 \\
\hline ARC & 3102 & 18,4 & 355871 \\
\hline
\end{tabular}

\footnotetext{
${ }^{14}$ Déperdition moyenne supérieure ou égale à 5 (sur une échelle de 6)
} 

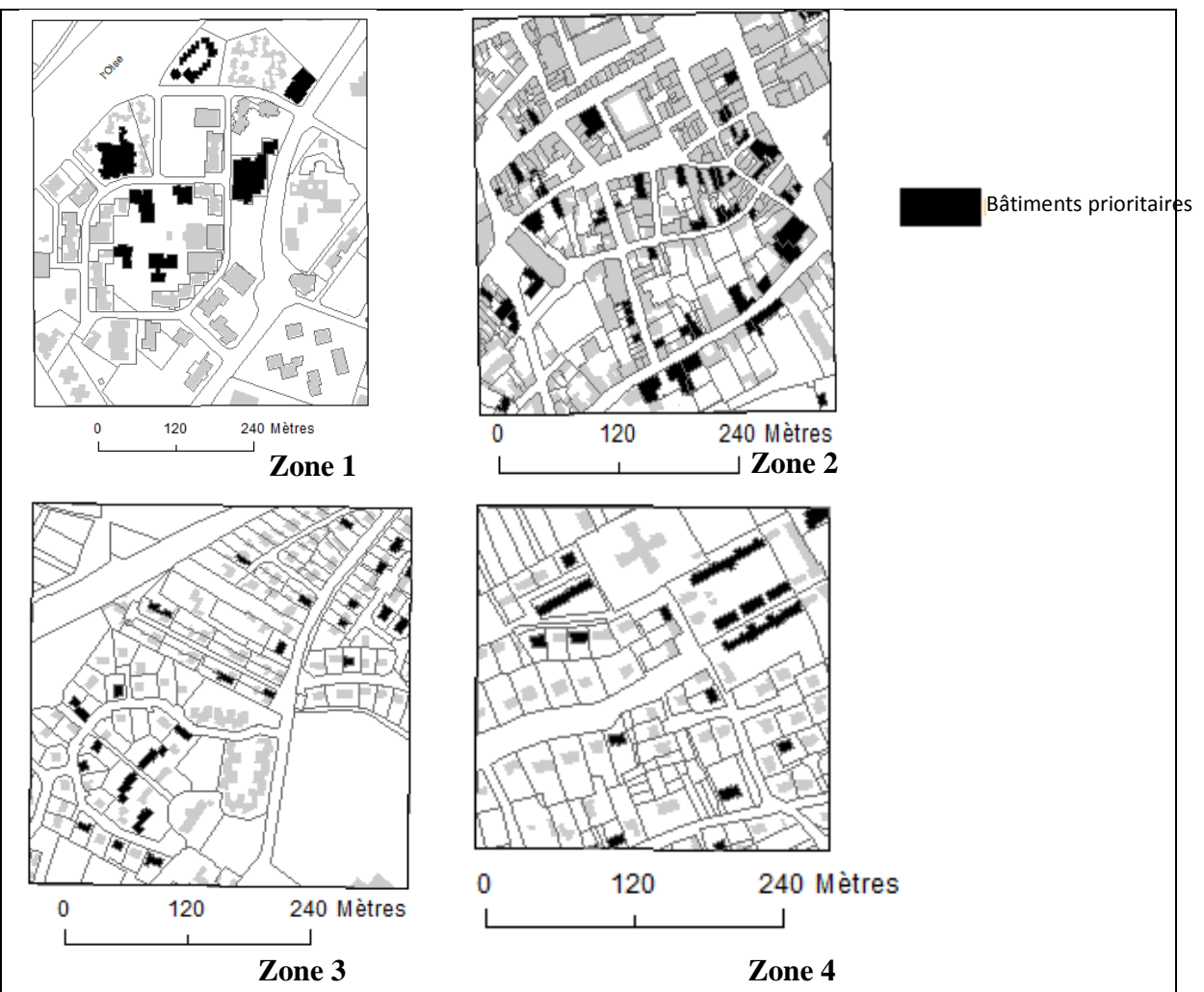

Figure 7 : Zones d'intervention prioritaires

\section{Interprétation}

Plus de $18 \%$ du parc résidentiel de l'ARC est concerné par cet indicateur. La zone 1, composée essentiellement de logements collectifs et d'un groupe scolaire, est principalement touchée puisque près de $36 \%$ de son parc est concerné (bâtiments de 5 étages et moins). Un travail d'information et d'accompagnement du ou des bailleurs sociaux concernés devra être mené en priorité.

\section{Limite de cet indice}

Cet indice ne tient cependant pas compte du profil socioéconomique des personnes logeant dans ces bâtiments. La qualité thermique d'un bâtiment et les faibles revenus de ses occupants sont deux des trois facteurs imbriqués qui conduisent à la précarité énergétique d'un ménage (Devalière, 2007 ; Réseau RAPPEL, 2011). En effet, des consommations énergétiques excessives, liées à une mauvaise isolation du logement, seront d'autant plus préjudiciables que les 
personnes concernées ont des revenus modestes. Dans certains cas, la mauvaise isolation du logement accroît donc le risque de précarité énergétique.

\section{4 : Délimitation des zones d'intervention ultra prioritaires}

Pour intégrer ce dernier paramètre, nous avons entrepris d'identifier les secteurs à risque de précarité énergétique. Nous avons ainsi exploité des données carroyées de l'INSEE $^{15}$ pour localiser les zones géographiques présentant un revenu moyen bas. Pour rappel, la notion de « revenu bas », telle que définie par l'INSEE, regroupe les ménages dont le revenu fiscal par unité de consommation se situe en dessous du seuil de «bas revenu» $(60 \% \text { de la médiane de la distribution })^{16}$. Nous avons considéré les zones à bas revenus comme celles ayant plus de $25 \%$ de leur ménage sous le seuil de bas revenus ${ }^{17}$. Les zones ainsi sélectionnées ont ensuite été croisées avec les bâtiments identifiés comme prioritaires.

\section{Interprétation}

Ce dernier indicateur restreint grandement le nombre de bâtiments prioritaires et nous concentre sur ce qu'on pourrait nommer les bâtiments ultra prioritaires. Ceuxci correspondent à moins de $2 \%$ du parc résidentiel de l'agglomération, mais souligne les inégalités territoriales. En effet, sur les quatre zones choisies : deux ne comportent aucun bâtiment ultra prioritaire, une est concernée de manière marginale (moins de $3 \%$ de son parc) et la dernière est concernée sur près de $37 \%$ de son parc (cf tableau 5 et figure 8). Dans la zone 1, les quatre bâtiments du groupe scolaire (cf figure 1) ressortent comme ultra prioritaires car ils font partie d'un carreau INSEE de bas revenu. Même s'ils ne sont pas, à proprement parler, concernés par un risque de précarité énergétique, cette information est importante pour l'ARC qui en tiendra compte pour la définition de nouvelles zones d'intervention à l'échelle d'un quartier.

Tableau 5 : Analyse statistique de l'indice 4

\begin{tabular}{|c|c|c|c|}
\hline Zone & $\begin{array}{c}\text { Nombre de bâtiments } \\
\text { «ultra prioritaires » }\end{array}$ & $\begin{array}{c}\% / \text { total parc } \\
\text { résidentiel de la zone }\end{array}$ & $\begin{array}{c}\text { Superficie } \\
\text { concernée }\left(\mathrm{m}^{2}\right)\end{array}$ \\
\hline 1 & 9 & 36,7 & 11722 \\
\hline 2 & 0 & 0 & 0 \\
\hline 3 & 0 & 0 & 0 \\
\hline 4 & 2 & 2,7 & 757 \\
\hline ARC & 184 & 1,4 & 26447 \\
\hline
\end{tabular}

\footnotetext{
${ }^{15} \mathrm{https} / / / \mathrm{www}$. insee.fr/fr/statistiques/2520034

${ }^{16} \mathrm{http} / / / \mathrm{www}$. insee.fr/fr/methodes/default.asp?page=definitions/pauvrete-monetaire.htm

${ }^{17}$ rassemble $10 \%$ de la population totale de l'ARC
} 

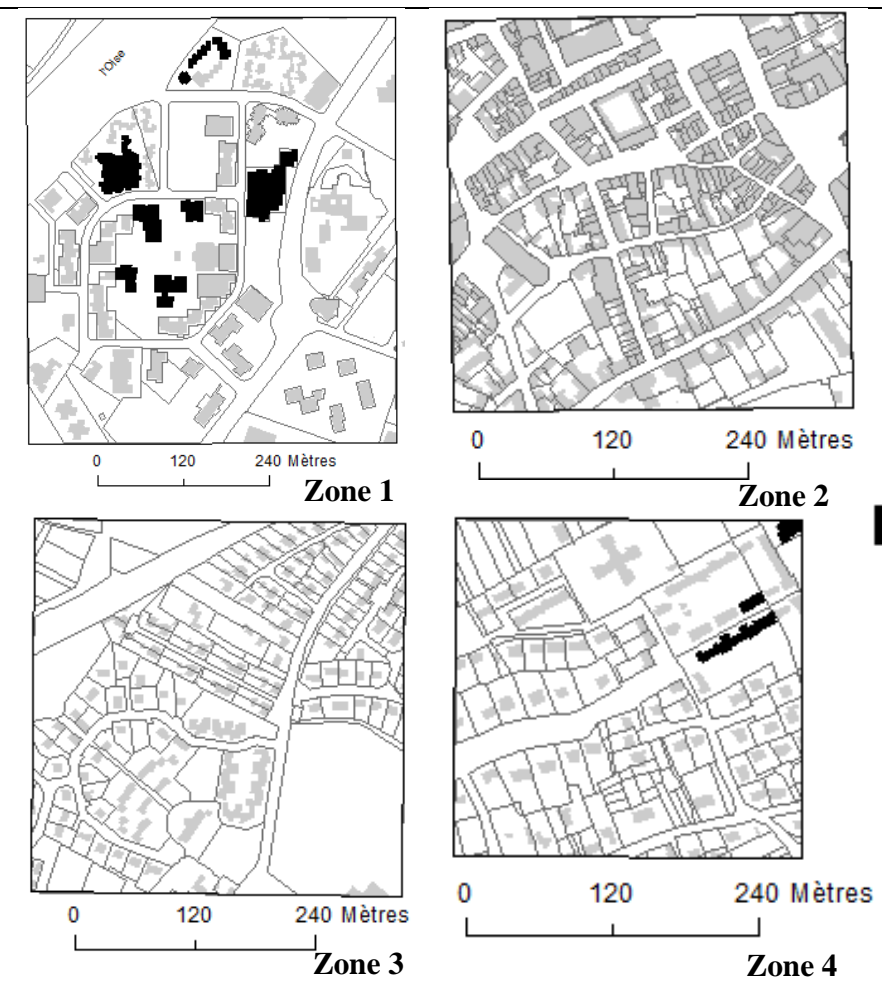

Bâtiments ultra prioritaires

Figure 8 : Zones d'intervention ultra prioritaires

Ce premier travail de localisation des zones d'intervention est une première étape d'investigation à l'échelle de l'agglomération. Elle devra être affinée grâce à des actions plus fines sur les bâtiments identifiés: thermographie de façades, interrogation des occupants de logements individuels pour approfondir l'interprétation de l'image thermographique.

La définition de ces zones prioritaires et ultra prioritaires va permettre à l'agglomération d'organiser ses actions :

- Envers les occupants et propriétaires de maisons individuelles et les occupants et copropriétaires de logements collectifs : informations sur les diagnostics à effectuer, les travaux envisageables, les possibilités de groupements d'achats et les aides financières existantes (ANAH, 2016) ; 
- Envers les bailleurs sociaux : sensibilisation, informations sur les diagnostics à effectuer, les travaux envisageables, les aides financières existantes $^{18}$;

- À son échelle, seule ou en collaboration avec des Agences Nationales spécialisées comme l'ADEME, l'ANRU ou l'ANAH, les services de l'ETA: mise en place de politiques publiques ciblées telles que des Opérations Programmées d'Amélioration de l'Habitat (OPAH), le PlanClimat-Energie Territoriaux (PCET).

\section{Conclusion}

Globalement, l'étude du parc résidentiel de l'Agglomération de la Région de Compiègne confirme selon nous la thermographie aérienne comme outil de sensibilisation, mais également comme outil opérationnel de diagnostic des performances énergétiques tant à l'échelle du bâtiment qu'à à l'échelle de la ville. En prenant bien en considération l'ensemble des limites liées à l'interprétation des cartes thermiques, il nous semble possible d'exploiter efficacement les données de déperditions énergétiques du parc résidentiel compiégnois en confrontant les résultats obtenus à l'indice de déperdition moyenne des bâtiments, au calcul de la part de la toiture dans cette déperdition, afin d'en informer les populations et les propriétaires privés ou bailleurs en charge du parc. Dès lors, l'étude menée a permis d'identifier des zones d'intervention prioritaires, liées à une forte déperdition énergétique, voire corrélées aux revenus moyens des populations.

Il est encore tôt, un an après le lancement de la campagne de sensibilisation, pour mesurer les retombées réelles de cette action. Les premiers retours sont plutôt encourageants $^{19}$ : plus de 5500 connexions uniques sur la page présentant les résultats de la thermographie 8 mois après sa mise en ligne, 650 familles sensibilisées lors des deux évènements organisés en septembre 2015 , le salon de restitution des résultats de la thermographie fin septembre 2015 ( 2 jours) et la foireexpo début octobre 2015 (4 jours), ainsi que près de 400 rendez-vous conseil pris avec le conseiller info-énergie entre octobre 2015 et novembre 2016 sont encourageants. L'ARC comptant 12000 bâtiments d'habitation, on peut estimer que près de $50 \%$ des ménages ont été sensibilisés.

En parallèle de cette campagne de sensibilisation et d'information individuelle, les deux outils créés dans le cadre de l'atelier-projet (analyse du parc bâti et base de

\footnotetext{
${ }^{18}$ Par exemple, l'éco-prêt logement social (éco-PLS) est un dispositif, issu du Grenelle de l'environnement, permettant la rénovation énergétique des 800000 logements sociaux les plus consommateurs en énergie, «logements énergivores », d'ici à 2020. Il s'agit d'un prêt accessible aux bailleurs sociaux.

${ }^{19}$ Sources ARC
} 
données sur la performance énergétique du parc public) vont permettre à l'ARC de cibler ses interventions et de mettre en place une politique publique qui lui donnera les moyens de diminuer ses émissions de gaz à effet de serre.

La thermographie aérienne permet ainsi d'aller au-delà d'une modélisation théorique de la performance énergétique d'un parc bâti. L'outil proposé dans le cadre de ce travail permet à une collectivité d'avoir rapidement une vue d'ensemble de son parc bâti. Cette première approche lui permet de localiser les zones sur lesquelles il sera nécessaire, dans un premier temps, d'effectuer des études plus fines et dans un deuxième temps, si les faits sont confirmés, mettre en place des actions de sensibilisation et d'information auprès des propriétaires, des occupants ou des bailleurs sociaux.

Pour aller plus loin, il serait intéressant de réfléchir à la manière d'améliorer la qualité des données caractérisant le parc bâti. Une comparaison de nos résultats avec ceux obtenus par une modélisation typologique serait également pertinente.

\section{Remerciements}

Nous remercions les élèves-ingénieurs en Génie des Systèmes Urbains pour avoir participé à l'élaboration de ce diagnostic territorial des déperditions énergétiques, les chargés de mission de l'ARC: Charlotte Kuzniac (Responsable $d u$ service environnement, Pôle Développement Durable), Florent Vanhoutte (Responsable du Service Information Géographique) et Armelle Gorgue (Chef de projet PLUi - Plan Local d'Urbanisme Intercommunal) pour cette commande à l'interface entre formes urbaines, politiques énergétique locale et habitat, ainsi que nos deux relecteurs anonymes pour les conseils nous ayant permis de tirer de ce cas exploratoire une analyse entre recherche et action territoriale.

\section{Bibliographie}

ADEME (2015). Isoler son logement, pour réduire les dépenses d'énergie et améliorer le confort d'un logement existant. Guide ADEME n`5614 juillet $201535 \mathrm{p}$. http://www.ademe.fr/sites/default/files/assets/documents/guide-pratique-isoler-sonlogement.pdf

Agence d'urbanisme Bordeaux Métropole Aquitaine (A’Urba) (2009). Caractérisation thermique du parc bâti résidentiel de la CUB. Rapport d'étude, $52 \mathrm{p}$.

Agence d'urbanisme Bordeaux Métropole Aquitaine (A’Urba) (2011). Rénovation thermique $d u$ parc bâti résidentiel de la CUB, Définition d'une méthodologie. Rapport méthodologique, $49 \mathrm{p}$.

Agence d'urbanisme Bordeaux Métropole Aquitaine (A’Urba) et Agence locale de l'énergie et du climat Métropole bordelaise et Gironde (Alec) (2013) Vers une planification énergétique "facteur 4 » de l'agglomération bordelaise : les grands principes. Rapport prospectif, $32 \mathrm{p}$.

Al-Homoud M.S. (2005), Performance characteristics and practical applications of common building thermal insulation materials, Building and Environment, vol. 40, pp. 353-366. 
Allinson, D. (2007). Evaluation of aerial thermography to discriminate loft insulation in residential housing, Thèse en philosophie, University of Nottingham, 208 p.

ANAH (2016). Les aides en pratique, $23 \mathrm{p}$ http://www.anah.fr/fileadmin/anah/Mediatheque/Publications/Les_aides/Anah_Les_aides _en_pratique.pdf

Arantes L., Marry S., Baverel O. et Quenard D. (2016). Efficacité énergétique et formes urbaines: élaboration d'un outil d'optimisation morpho-énergétique, Cybergeo : European Journal of Geography http://cybergeo.revues.org/27584; DOI : $10.4000 /$ cybergeo. 27584

Baker N., Steemers K. (2000), Energy and environment in architecture: a technical design guide, New-York, E\&FN. DOI : 10.4324/9780203223017

Bertrand F., Rocher L. (2011). L'intégration du changement climatique dans l'action publique locale, facteur de renouvellement du développement urbain durable?, dans Béal V., Gauthier M., Pinson G., Le développement durable changera-t-il la ville ? Le regard des sciences sociales, Presse universitaires de Saint Etienne, pp. 385-403.

Bertrand F., Richard E. (2014). L'action des collectivités territoriales face au «problème climat» en France : une caractérisation par les politiques environnementales, Natures Sciences Sociétés 3/2014 (Vol. 22), p. 195-203, www.cairn.info/revue-natures-sciencessocietes-2014-3-page-195.htm. DOI : 10.1051/nss/2014036.

Chanard C. (2011). Territoire et énergie: politiques locales, échelles d'intervention et instruments de mobilisation, de connaissance et d'action, Thèse de doctorat en Géographie, Université de Franche-Comté, 309 p.

Chanard C., de Sède-Marceau M.-C.et Robert M. (2011), « Politique énergétique et facteur 4 : instruments et outils de régulation à disposition des collectivités », Développement durable et territoires [En ligne], Vol. 2, $\mathrm{n}^{\circ} 1 \mid$ Mars 2011.

http://developpementdurable.revues.org/8776 ; DOI :10.4000/developpementdurable.8776

Chandelier L., Bur G., Bouffier J. (2014). Contributions des technologies satellitaires à l'étude de îlots de chaleur urbains, Actes du $12^{e}$ Séminaire annuel de l'observation urbaine: Observer l'environnement en ville, ressources et nuisances, http://www.territoires-ville.cerema.fr/IMG/pdf/17_L_CHANDELIER_cle2edfc7-1.pdf

Debizet G. (2013). Représentation de l'ilot de Chaleur Urbain : limites de la thermographie aérienne et raisons de son succès. Colloque Territoires vulnérables, villes résilientes: quelle adaptation possible dans le contexte des changements climatiques ?, Dec 2013, Montréal, Canada.

Devalière I. (2007). Comment prévenir la précarité énergétique ? Situation actuelle et risques inhérents à la libéralisation du service de l'énergie, Les annales de la recherche urbaine, $\mathrm{n}^{\circ} 103$, pp. 137-143.

Godinot S. (2011) Les plans climat énergie territoriaux : voies d'appropriation du facteur 4 par les collectivités et les acteurs locaux ? Développement durable et territoires [En ligne], Vol. 2, $\mathrm{n}^{\circ} 1 \mid$ Mars 2011.

https://developpementdurable.revues.org/8874. 
Ham Y., Golparvar-FardHay M. (2013). An automated vision-based method for rapid 3D energy performance modeling of existing buildings using thermal and digital imagery, Advanced Engineering Informatics, volume 27, issue 3, Pages 395-409

Hay G.J., Kyle C.D., Hemachandran B., Chen G., Rahman M.M., Fung T.S., and Arvai J.L. (2011). "Geospatial Technologies to Improve Urban Energy Efficiency". Remote Sensing 3, n 7, pp. 1380-1405.

Henry JB, Pierrard S., Ridoux P. (2009). Thermographie aérienne des Villes, Mythes et réalités, Revue $X Y Z \mathrm{n}^{\circ} 118$, pp 39-42.

Kavgica M., Mavrogiannia A. Mumovica D., Summerfieldb A., Stevanovicc Z., DjurovicPetrovicd M. (2010). A review of bottom-up building stock models for energy consumption in the residential sector, Building and Environment, Volume 45, Issue 7, pp 1683-1697.

LNE (Laboratoire National de Métrologie et d'Essais), (2015). Comprendre les images de thermographie infrarouge aérienne, document de formation «experte» préparé par Véronique Le Sant pour le département de télédétection du LNE.

Lefebvre M. (2008). Télédétection et cartographie : des outils au service de la thermographie aérienne, revue $X Y Z, \mathrm{n}^{\circ} 116$, pp 52-57.

Maïzia M., (2007). L'énergétique urbaine et la morphologie des villes, Les annales de la recherche urbaine, $\mathrm{n}^{\circ} 103$, pp. 79-85.

Maresca B., Dujin A., Picard R. (2009). La consommation d'énergie dans l'habitat entre recherche de confort et impératif écologique, Cahier de recherches $\mathrm{n}^{\circ} 264$, CREDOC, $87 \mathrm{p}$.

MEDDAT (2009)., Diagnostic de performances énergétiques : Guide de recommandations, $71 \mathrm{p}$.

MEDDE, (2013). Le facteur 4 en France: la division par 4 des émissions de gaz à effet de serre à l'horizon 2050, Rapport final, http://www.cgedd.developpementdurable.gouv.fr/IMG/pdf/008378-01_rapport-final_cle0aca84.pdf

Poupeau, F.-M., (2013). Quand l'État territorialise la politique énergétique. L'expérience des schémas régionaux du climat, de l'air et de l'énergie, Politiques et management public [En ligne], Vol 30/4 |2013, mis en ligne le 09 avril 2016, consulté le 24 septembre 2016. URL : http://pmp.revues.org/6830

Ratti C., Baker N., Steemers K., (2005). Energy consumption and urban texture, Energy and Buildings, Vol.37, $\mathrm{n}^{\circ}$ 7, pp. 762-776. DOI : 10.1016/j.enbuild.2004.10.010

Réseau RAPPEL (2001). Précarité énergétique : état des lieux et propositions d'actions, janvier 2011, http://www.precarite-energie.org/IMG/pdf/Precarite_energetique__tables_rondes_departementales_V2-2.pdf

Theys J. et Vidalenc É. (2011). Le territoire, un levier complémentaire pour l'atteinte du facteur 4, Développement durable et territoires [En ligne], Vol. 2, $\mathrm{n}^{\circ} 1 \mid$ Mars 2011, mis en ligne le 15 janvier 2013, consulté le 25 septembre 2016. http://developpementdurable.revues.org/8748, DOI : 10.4000/developpementdurable.8748

Vellico M., Blanos R., Coren F. (2011). Thermal images processing for energy efficiency evaluation in buildings, 17th Building Services Mechanical and Building Industry Days, Urban energy conference, pp. 43-47. 
Apports et limites de la thermographie 39

Vermeulen T., Kämpf J.H., Beckers B. (2013). Urban Form Optimization for the Energy Performance of Buildings Using Citysim, Proceedings of CISBAT 2013 Cleantech for Smart Cities and Buildings, vol. II, pp. 915-920.

Zayane C. (2011). Identification d'un modèle de comportement thermique de bâtiment à partir de sa courbe de charge. Thèse. Ecole Nationale Supérieure des Mines de Paris, $135 \mathrm{p}$.

Article reçu le : AR_1religne_soumission

Article accepté le : AR_soumission 